Mariëtte B. van Veenendaal Martijn Miedema

Frans H. C. de Jongh

Johanna $H$. van der Lee

Inez Frerichs

Anton H. van Kaam

\section{Effect of closed endotracheal suction in high-frequency ventilated premature infants measured with electrical impedance tomography}

Received: 16 March 2009

Accepted: 7 September 2009

Published online: 23 September 2009

(C) The Author(s) 2009. This article is published with open access at Springerlink.com

M. B. van Veenendaal · M. Miedema . F. H. C. de Jongh - A. H. van Kaam (-) Department of Neonatology (H3-144), Emma Children's Hospital AMC, P.O. Box 22660, 1100 DD Amsterdam, The Netherlands

e-mail: a.h.vankaam@amc.uva.nl

Tel.: +31-20-5669111

Fax: +31-20-6965099

J. H. van der Lee

Pediatric Clinical Epidemiology, Emma Children's Hospital AMC, Amsterdam, The Netherlands

I. Frerichs

Department of Anaesthesiology and Intensive Care Medicine, University Medical Centre Schleswig-Holstein, Campus Kiel, Kiel, Germany
Abstract Objective: To determine the global and regional changes in lung volume during and after closed endotracheal tube (ETT) suction in high-frequency ventilated preterm infants with respiratory distress syndrome (RDS). Design: Prospective observational clinical study. Set-

ting: Neonatal intensive care unit. Patients: Eleven non-muscle relaxed preterm infants with RDS ventilated with open lung high-frequency ventilation (HFV).

Interventions: Closed ETT suction. Measurements and results: Changes in global and regional lung volume were measured with electrical impedance tomography. ETT suction resulted in an acute loss of lung volume followed by spontaneous recovery with a median residual loss of $3.3 \%$ of the maximum volume loss. The median stabilization time was $8 \mathrm{~s}$. At the regional level, the lung volume changes during and after ETT suction were heterogeneous in nature. Conclusions: Closed ETT suction causes an acute, transient and heterogeneous loss of lung volume in premature infants with RDS treated with open lung HFV.

Keywords Endotracheal suction · Premature infant · High-frequency ventilation - Electrical impedance tomography

\section{Introduction}

Bronchopulmonary dysplasia (BPD) is a common complication of premature birth, and (regional) alveolar collapse and overdistension during mechanical ventilation are considered important risk factors [1]. In an attempt to reduce the incidence of BPD in preterm infants, highfrequency ventilation (HFV), using a so-called open lung ventilation strategy aiming to minimize alveolar collapse and overdistension, has been advocated $[2,3]$.
Endotracheal tube (ETT) suction is essential in ventilated patients to maintain airway patency. However, ETT suction may also lead to (transient) hypoxia and cardiovascular instability, side effects generally attributed to loss of lung volume due to atelectasis [4-6]. This increased risk of atelectasis during ETT suction may compromise the efficacy of open lung ventilation to attenuate lung injury.

Only two studies have explored the effect of ETT suction on lung volume in newborn infants on HFV, using 
respiratory inductive plethysmography (RIP) [7, 8]. However, these studies included relatively mature, and in some cases muscle relaxed, newborn infants ventilated for various causes of respiratory failure. The results may be different in non-muscle relaxed preterm infants with respiratory distress syndrome (RDS). Furthermore, RIP only measures changes in total lung volume, providing no information on regional atelectasis and overdistension.

Electrical impedance tomography (EIT) is a new noninvasive technique measuring both global and regional changes in lung impedance, which are highly correlated with changes in gas volume [9]. The aim of this study was to examine the effect of closed ETT suction on the relative changes in both global and regional lung impedance in preterm infants with RDS treated with open lung HFV.

\section{Methods}

\section{Subjects}

The study was performed in the neonatal intensive care unit of the Emma Children's Hospital AMC in Amsterdam and approved by the Institutional Review Board. Premature infants with RDS treated with primary HFV were included after obtaining written informed consent from both parents.

HFV was delivered with a Sensormedics 3100A oscillator (Cardinal Health, Yorba Linda, CA) and at all times combined with an individualized open lung ventilation strategy aiming to recruit and stabilize collapsed alveoli with the lowest possible airway pressure [2].

\section{Suction protocol}

ETT suction, using a neonatal closed tracheal suction system (Trach Care, Ballard Medical Products, Draper, UT), was performed in supine position with the patient's head in the midline. Catheter sizes were standardized according to ETT size. Following saline $(0.3 \mathrm{ml})$ instillation, the catheter was inserted to the tip of the ETT, and suctioning was applied for approximately $3 \mathrm{~s}$ at $-75 \mathrm{mmHg}$. Next, the catheter was withdrawn while continuing suctioning.

EIT measurements and analysis

Changes in lung impedance and airway pressures were continuously recorded from $60 \mathrm{~s}$ before until $120 \mathrm{~s}$ after ETT suction with the "Goettingen Goe-MF II" EIT system (Cardinal Health, Yorba Linda, CA) using an electrical current of $5 \mathrm{~mA}_{\mathrm{rms}}(50 \mathrm{kHz})$ and a scan rate of $44 \mathrm{~Hz}[10]$.

EIT data were analyzed off-line. Each recording was divided into three phases: (1) pre-suction (baseline), defined as -40 to $-10 \mathrm{~s}$ before starting ETT suction; (2) suction, defined as the period in which negative pressure was applied; (3) post-suction, defined as +5 to $+30 \mathrm{~s}$ after stabilization of the EIT signal. If the EIT signal was unstable, a stable time period was selected as close as possible to the predefined time windows. The post-suction EIT signal was considered stabilized when no visual increases were seen at the peaks and troughs of the signal.

Using the pre-suction phase as the reference recording, the mean global change in lung impedance after ETT suction was calculated by averaging all unfiltered impedance values. We also determined the lowest impedance level in the suction phase. With these values we could determine the residual global loss of impedance after suction as a percentage of the maximum global loss during suction. We also calculated the maximum and residual impedance loss in four regions of interest (ROI), i.e., ventral, dorsal, left and right. The time necessary to recover $80 \%$ of the maximum loss from the time point of

Table 1 Patient characteristics

\begin{tabular}{|c|c|c|c|c|c|c|c|c|c|c|}
\hline $\begin{array}{l}\text { Infant } \\
\text { no. }\end{array}$ & Diagnosis & $\begin{array}{l}\text { GA } \\
\text { (weeks) }\end{array}$ & $\begin{array}{l}\text { Weight } \\
\text { (kg) }\end{array}$ & $\begin{array}{l}\text { Age } \\
(\mathrm{h})\end{array}$ & $\begin{array}{l}\text { ETT size } \\
(\mathrm{mm})\end{array}$ & $\begin{array}{l}\text { Cat } \\
\text { size (Fr) }\end{array}$ & $\begin{array}{l}\text { Area }^{\mathrm{a}} \\
\left(\mathrm{mm}^{2}\right)\end{array}$ & $\begin{array}{l}\mathrm{CDP} \\
\left(\mathrm{cmH}_{2} \mathrm{O}\right)\end{array}$ & $\begin{array}{l}\text { Amplitude } \\
\left(\mathrm{cmH}_{2} \mathrm{O}\right)\end{array}$ & $\mathrm{FiO}_{2}$ \\
\hline 1 & RDS & 25.3 & 0.83 & 14 & 3.0 & 6 & 4.0 & 14 & 25 & 0.25 \\
\hline 2 & RDS & 27.3 & 0.98 & 3 & 3.0 & 6 & 4.0 & 14 & 18 & 0.21 \\
\hline 3 & $\mathrm{RDS}+\mathrm{PPHN}$ & 29.3 & 1.37 & 45 & 3.5 & 7 & 5.3 & 12 & 24 & 0.80 \\
\hline 4 & RDS & 31.1 & 1.44 & 48 & 3.0 & 6 & 4.0 & 9 & 19 & 0.22 \\
\hline 5 & RDS & 33.6 & 2.12 & 71 & 3.0 & 6 & 4.0 & 8 & 24 & 0.25 \\
\hline 6 & RDS & 28.3 & 1.30 & 9 & 3.0 & 6 & 4.0 & 18 & 29 & 0.24 \\
\hline 7 & RDS & 31.4 & 1.39 & 9 & 3.5 & 7 & 5.3 & 18 & 22 & 0.30 \\
\hline 8 & RDS + PPHN & 31.4 & 1.83 & 33 & 3.5 & 7 & 5.3 & 14 & 28 & 0.55 \\
\hline 9 & RDS & 25.6 & 0.79 & 10 & 2.5 & 5 & 2.7 & 16 & 22 & 0.30 \\
\hline 10 & RDS + PPHN & 31.0 & 1.60 & 36 & 3.0 & 6 & 4.0 & 13 & 21 & 0.60 \\
\hline 11 & RDS & 28.4 & 1.12 & 7 & 3.0 & 6 & 4.0 & 18 & 25 & 0.35 \\
\hline Median & & 29.3 & 1.37 & 14 & 3.0 & 6 & 4.2 & 14 & 24 & 0.30 \\
\hline
\end{tabular}

GA Gestational age, ETT endotracheal tube, Cat suction catheter, a Unoccluded cross-sectional area of the ETT during suctioning $\mathrm{CDP}$ continuous distending pressure, $\mathrm{FiO}_{2}$ fraction of inspired oxygen, $R D S$ respiratory distress syndrome, $P P H N$ persistent pulmonary hypertensions of the newborn 
maximum impedance loss and the time necessary for the EIT signal to stabilize after ETT suction was stopped were calculated.

Data collection

We collected the following parameters: transcutaneous oxygen saturation $\left(\mathrm{SpO}_{2}\right)$ before, during and after ETT suction, episodes of bradycardia (heart rate below $100 \mathrm{bpm}$ ) during ETT suction and ventilator settings at the start of ETT suction. Adjustments in ventilator settings during the study were recorded.

\section{Statistical analysis}

Data are presented as median and interquartile range (IQR). Differences in $\mathrm{SpO}_{2}$ before and during ETT suction were analyzed with the Wilcoxon signed ranks test. A $P$ value less than 0.05 is considered significant.

\section{Results}

\section{Patient characteristics}

Twelve patients were studied. One patient had to be excluded because the EIT signal before suction was not stable. Table 1 shows the characteristics of the remaining 11 patients. The indication for ETT suction was either routine suctioning before surfactant administration $(n=10)$ or clinical signs of secretion in the upper airways and/or the ETT $(n=1)$.

\section{EIT results}

In all patients ETT suction resulted in a steep reduction of global lung impedance followed by a more gradual recovery once suctioning was stopped (Fig. 1, Table 2). Comparing the ROI showed that the distribution of the maximum impedance loss was heterogeneous in nature in almost all patients and that there was no consistent pattern favoring one of these regions.

In all patients the global impedance stabilized within $60 \mathrm{~s}$ after suctioning, with a median stabilization time of $8 \mathrm{~s}$ (IQR 3-29 s). The median time needed to recover $80 \%$ of the maximum loss from the time at which this maximum volume loss occurred was $8 \mathrm{~s}$ (IQR 4-14 s).

In 7 of the 11 patients (64\%) global impedance stabilized below pre-suction values, and 3 patients failed to ever recover $90 \%$ of the impedance lost during suctioning (Table 2). In the remaining patients global impedance
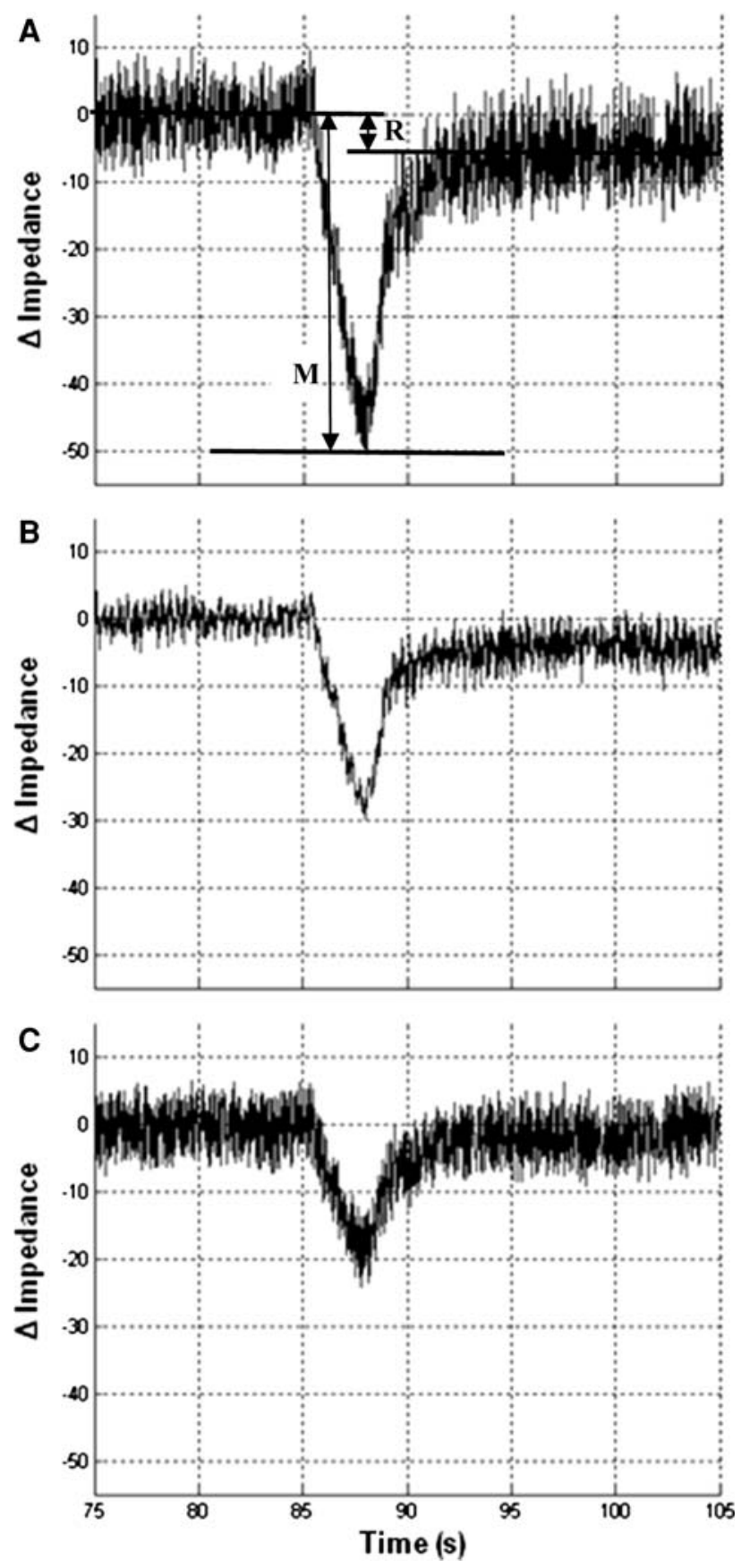

Fig. 1 Representative tracing of global (a), ventral (b) and dorsal (c) impedance changes during endotracheal tube suction (infant 1). The lines in a indicate the mean pre-suction impedance, the maximum negative impedance level during suctioning and the mean post-suction impedance after stabilization. With this information the maximum impedance loss $(M)$ and the residual impedance loss after suctioning $(R)$ are calculated

returned to baseline $(n=2)$ or stabilized at higher levels $(n=2)$. The median residual loss in global impedance after stabilization for all 11 patients was $3.3 \%$ of the 
Table 2 Changes in impedance during and after closed endotracheal tube suction compared to the baseline (zero) values

\begin{tabular}{|c|c|c|c|c|c|c|c|c|c|c|}
\hline \multirow[t]{3}{*}{ Infant no. } & \multicolumn{5}{|c|}{ Maximum impedance loss during suction } & \multicolumn{5}{|c|}{ Residual impedance loss after suction } \\
\hline & \multirow[t]{2}{*}{$\begin{array}{l}\text { Global } \\
\Delta \mathrm{Z}\end{array}$} & \multicolumn{4}{|c|}{$\begin{array}{l}\text { Region of interest } \\
\text { (\% of maximum global loss) }\end{array}$} & \multirow[t]{2}{*}{$\begin{array}{l}\text { Global } \Delta \mathrm{Z} \\
\text { (\% of max loss) }\end{array}$} & \multicolumn{4}{|c|}{$\begin{array}{l}\text { Region of interest } \\
\text { (\% of residual global loss) }\end{array}$} \\
\hline & & $\mathrm{V}$ & $\mathrm{D}$ & $\mathrm{R}$ & $\mathrm{L}$ & & $\mathrm{V}$ & $\mathrm{D}$ & $\mathrm{R}$ & $\mathrm{L}$ \\
\hline 1 & -51 & $-31(59)$ & $-20(41)$ & $-24(48)$ & $-26(52)$ & $-5.1(10)$ & $-4.5(88)$ & $-0.6(12)$ & $-3.7(72)$ & $-1.5(28)$ \\
\hline 2 & -68 & $-39(57)$ & $-29(43)$ & $-33(48)$ & $-35(52)$ & $-2.3(3)$ & $-2.3^{\mathrm{a}}$ & $0.07^{\mathrm{a}}$ & $-1.2(51)$ & $-1.1(49)$ \\
\hline 3 & -43 & $-15(35)$ & $-28(65)$ & $-24(55)$ & $-19(45)$ & $6.2^{\mathrm{b}}$ & $3.7(60)$ & $2.5(40)$ & $2.1(34)$ & $4.1(66)$ \\
\hline 4 & -62 & $-31(50)$ & $-31(50)$ & $-35(57)$ & $-27(43)$ & $-1.8(3)$ & $1.9^{\mathrm{a}}$ & $-3.7^{\mathrm{a}}$ & $-1.4(76)$ & -0.4 \\
\hline 5 & -58 & $-41(70)$ & $-17(30)$ & $-27(46)$ & $-31(54)$ & $0.02(0)$ & $0.5^{\mathrm{a}}$ & $-0.4^{\mathrm{a}}$ & $-1.3^{\mathrm{a}}$ & $1.3^{\mathrm{a}}$ \\
\hline 6 & -67 & $-31(46)$ & $-36(54)$ & $-46(69)$ & $-21(31)$ & $-0.1(0.2)$ & $-0.3^{\mathrm{a}}$ & $0.2^{\mathrm{a}}$ & $-1.2^{\mathrm{a}}$ & $1.1^{\mathrm{a}}$ \\
\hline 7 & -34 & $-17(50)$ & $-17(50)$ & $-19(57)$ & $-15(43)$ & $-3.7(11)$ & $-0.3(7)$ & $-3.4(92)$ & $-2.4(65)$ & $-1.3(35)$ \\
\hline 8 & -40 & $-22(55)$ & $-18(45)$ & $-21(52)$ & $-19(48)$ & $-3.5(9)$ & $-2.8(81)$ & -0.7 (19) & $-0.8(23)$ & $-2.7(77)$ \\
\hline 9 & -59 & $-35(60)$ & $-24(40)$ & $-31(52)$ & $-28(48)$ & $-8.7(15)$ & $-3.4(39)$ & $-5.3(61)$ & $-4.1(47)$ & $-4.6(53)$ \\
\hline 10 & -49 & $-25(51)$ & $-24(49)$ & $-24(49)$ & $-25(51)$ & $-6.6(13)$ & $-4.2(63)$ & $-2.4(37)$ & $-4.0(61)$ & $-2.6(39)$ \\
\hline 11 & -48 & $-32(66)$ & $-16(34)$ & $-27(56)$ & $-21(44)$ & $9.3^{\mathrm{b}}$ & $2.3(25)$ & $7.0(75)$ & $3.5(37)$ & $5.8(63)$ \\
\hline Median & -51 & $-31(55)$ & $-24(45)$ & $-27(52)$ & $-25(48)$ & -2.3 & -0.3 & -0.6 & -1.3 & -1.1 \\
\hline
\end{tabular}

$V$ Ventral, $D$ dorsal, $R$ right, $L$ left

a Regions with volume loss and increase after endotracheal suction

b Impedance stabilized at higher level after suction compared to (percentages not shown because of the opposite regional values)

maximum loss during suction (IQR 0-11\%). Again, the loss of impedance after stabilization showed an inconsistent and heterogeneous pattern across the different ROIs.

\section{Physiological data}

ETT suctioning resulted in a transient decrease in the median $\mathrm{SpO}_{2}$ from 95\% (IQR 94-98\%) to 90\% (IQR 89$94 \%)(P<0.01)$. Bradycardia was not observed in any of the patients. No adjustments were made in the ventilator settings. In the majority of infants spontaneous breathing was absent or limited to just a few breaths per minute.

\section{Discussion}

This study shows that closed ETT suction during open lung HFV in non-muscle relaxed preterm infants with RDS results in an acute and heterogeneous loss of impedance followed by a gradual spontaneous recovery within the first minute after suctioning. We did not observe major hypoxia or cardiovascular instability.

Animal and human adult studies have shown that the changes in lung impedance during mechanical ventilation are highly correlated with changes in air content seen on computed tomography $[9,11,12]$. Although comparable studies in newborn infants are difficult to perform and therefore currently not available, it seems plausible that these observations also apply to ventilated newborn infants and that the observed changes in lung impedance as reported in the present study are indeed changes in lung aeration.

Our observed changes in global lung volume are strikingly similar to previous studies using RIP to measure changes in lung volume following ETT suction in more mature and, in some cases, muscle relaxed newborn infants ventilated for various causes of respiratory failure [7, 8]. At first glance, these consistent results seem to indicate that atelectasis caused by ETT suctioning during open lung HFV is only transient and that a recruitment maneuver to restore lung volume and attenuate possible lung injury is often not necessary. However, unlike RIP, EIT also provides important information on the regional distribution. To our knowledge, this is the first study that shows that the distribution of lung volume changes after ETT suction in ventilated preterm infants with RDS is heterogeneous in nature. Similar heterogeneity following ETT suction has also been described in children with acute respiratory distress syndrome [13].

Although our study cannot measure the exact volume states of different lung regions, our findings do suggest that recovery in global lung volume after ETT suctioning may still be accompanied by regional atelectasis and over-distension and thus ongoing lung injury. It might well be that these patients can indeed benefit from a recruitment maneuver aiming to reestablish the homogeneity in regional air distribution. Future studies will have to investigate the efficacy of such a maneuver.

It was interesting to observe that patients with the largest tube size showed the smallest decrease in lung volume during ETT suctioning. Although this could be explained by the larger unoccluded cross-sectional area of the ETT in these patients [14], the small sample size of this study precludes reliable analysis and thus firm conclusions.

This study has several limitations that need to be addressed. First of all, this study does not provide absolute lung volume changes in response to ETT suction. 
Because the main focus of this study was to determine the relative changes in lung volume following ETT suction, we decided not to subject the patients to a period of possibly injurious positive pressure ventilation with the sole purpose of calibrating the impedance changes. Secondly, this study mainly included surfactant-deficient preterm infants. The result may be different in other groups of patients, although the consistency of our findings with previous studies using RIP seems reassuring. Finally, the effect of ETT suction on lung volume may vary according to the suction protocol.

\section{Conclusion}

Closed ETT suction causes an acute and heterogeneous loss of lung volume in non-muscle relaxed premature infants with RDS treated with open lung HFV. Although there is an almost complete spontaneous recovery of the global volume loss within the first minute after ETT suction, at the regional level this recovery is heterogeneous in nature.

Acknowledgments This work was financially supported by Cardinal Health Care and Nycomed, The Netherlands.

Conflict of interest statement The authors have not disclosed any potential conflicts of interest.

Open Access This article is distributed under the terms of the Creative Commons Attribution Noncommercial License which permits any noncommercial use, distribution, and reproduction in any medium, provided the original author(s) and source are credited.

\section{References}

1. Clark RH, Slutsky AS, Gerstmann DR (2000) Lung protective strategies of ventilation in the neonate: what are they? Pediatrics 105:112-114

2. de Jaegere A, van Veenendaal MB, Michiels A, van Kaam AH (2006) Lung recruitment using oxygenation during open lung high-frequency ventilation in preterm infants. Am J Respir Crit Care Med 174:639-645

3. Tingay DG, Mills JF, Morley CJ, Pellicano A, Dargaville PA (2006) The deflation limb of the pressure-volume relationship in infants during highfrequency ventilation. Am J Respir Crit Care Med 173:414-420

4. Choong K, Chatrkaw P, Frndova H, Cox PN (2003) Comparison of loss in lung volume with open versus in-line catheter endotracheal suctioning. Pediatr Crit Care Med 4:69-73

5. Maggiore SM, Lellouche F, Pigeot J, Taille S, Deye N, Durrmeyer X, Richard JC, Mancebo J, Lemaire F, Brochard L (2003) Prevention of endotracheal suctioning-induced alveolar derecruitment in acute lung injury. Am J Respir Crit Care Med 167:1215-1224
6. Cereda M, Villa F, Colombo E, Greco G, Nacoti M, Pesenti A (2001) Closed system endotracheal suctioning maintains lung volume during volumecontrolled mechanical ventilation. Intensive Care Med 27:648-654

7. Hoellering AB, Copnell B, Dargaville PA, Mills JF, Morley CJ, Tingay DG (2008)

Lung volume and cardiorespiratory changes during open and closed endotracheal suction in ventilated newborn infants. Arch Dis Child Fetal Neonatal Ed 93:F436-F441

8. Tingay DG, Copnell B, Mills JF, Morley CJ, Dargaville PA (2007) Effects of open endotracheal suction on lung volume in infants receiving HFOV. Intensive Care Med 33:689-693

9. Victorino JA, Borges JB, Okamoto VN Matos GF, Tucci MR, Caramez MP, Tanaka H, Sipmann FS, Santos DC, Barbas CS, Carvalho CR, Amato MB (2004) Imbalances in regional lung ventilation: a validation study on electrical impedance tomography. Am J Respir Crit Care Med 169:791-800

10. Frerichs I, Schiffmann H, Hahn G, Hellige G (2001) Non-invasive radiation-free monitoring of regional lung ventilation in critically ill infants. Intensive Care Med 27:1385-1394
11. Frerichs I, Hinz J, Herrmann P, Weisser G, Hahn G, Dudykevych T, Quintel M, Hellige G (2002) Detection of local lung air content by electrical impedance tomography compared with electron beam CT. J Appl Physiol 93:660-666

12. Meier T, Luepschen H, Karsten J, Leibecke T, Grossherr M, Gehring H, Leonhardt S (2008) Assessment of regional lung recruitment and derecruitment during a PEEP trial based on electrical impedance tomography. Intensive Care Med 34:543-550

13. Wolf GK, Grychtol B, Frerichs I, van Genderingen HR, Zurakowski D, Thompson JE, Arnold JH (2007) Regional lung volume changes in children with acute respiratory distress syndrome during a derecruitment maneuver. Crit Care Med 35:19721978

14. Kiraly NJ, Tingay DG, Mills JF, Morley CJ, Copnell B (2008) Negative tracheal pressure during neonatal endotracheal suction. Pediatr Res 64:29-33 